\title{
Lista sistemática de la ictiofauna de Bahía de Banderas, México
}

\author{
A fish checklist for the ichthyofauna of Bahía de Banderas, Mexico
}

\author{
Rodrigo Moncayo-Estrada ${ }^{1}$, José Luis Castro-Aguirre ${ }^{2 *}$ y José De La Cruz-Agüero ${ }^{2}$ \\ ${ }^{1}$ Centro Interdisciplinario de Investigación para el Desarrollo Regional Integral, Unidad Michoacán, Instituto Politécnico Nacional (CIIDIR-IPN- \\ MICHOACÁN). Justo Sierra \# 28, Col. Centro, Jiquilpan, Michoacán, C. P. 59510. \\ ${ }^{2}$ Centro Interdisciplinario de Ciencias Marinas, Colección Ictiológica, Instituto Politécnico Nacional (CICIMAR-IPN). Ave. Instituto Politécnico Nacional \\ No. 1, Col. Playa Palo de Sta. Rita, La Paz, Baja California Sur 23096, México. \\ *Correspondencia: jlcastroaguirre@hotmail.com
}

\begin{abstract}
Resumen: La Bahía de Banderas ubicada en el Pacífico central mexicano alberga una gran riqueza íctica la cual se menciona por primera vez en este elenco sistemático, donde se incluyen 210 especies, 145 géneros y 74 familias. La lista se integra con representantes de 205 especies capturados durante recolectas realizadas entre 1997 a 1998, así como de otras cinco observadas e identificadas de modo positivo, aunque no recolectadas. Los resultados indican un número bastante alto con afinidad tropical (117) respecto a las templadas (7), y el resto (76) manifiestan una distribución muy amplia en el océano Pacífico oriental. Las familias mejor representadas, por su número de especies, son: Carangidae (19), Haemulidae (16), Sciaenidae (13), Serranidae (11) y Paralichthyidae (8). De acuerdo con el análisis de bibliografía actualizada, 11 amplían su ámbito de distribución conocida a Bahía de Banderas.
\end{abstract}

Palabras clave: ictiofauna, Bahía de Banderas, zoogeografía, ampliación de distribución.

\begin{abstract}
The Bahía de Banderas, located in the central Mexican Pacific, has a large diversity of fish species, which is described for the first time in the present systematic checklist, which includes a total of 210 species, belonging to 145 genera and 74 families. 205 species were collected between 1997 and 1998, and the remaining five were observed and positively identified but not captured. The results show an important tropical component (117) over the temperate one (7), and the rest of the species (76) with a wide distribution along the eastern Pacific Ocean. The best represented families by number of species were: Carangidae (19), Haemulidae (16), Sciaenidae (13), Serranidae (11) and Paralichthyidae (8). Accordingly to recent bibliographic records, eleven species reported here have extended their known distribution range to Bahía de Banderas.
\end{abstract}

Key words: icthyofauna, Banderas bay, zoogeography, range extension.

\section{Introducción}

Las investigaciones ictiológicas sobre la taxonomía alfa del Pacífico mexicano se han llevado a cabo en varias localidades, tanto del noroeste (Ramírez y Arvizu, 1965; Castro-Aguirre et al., 1970; van der Heiden y Findley, 1988; De La Cruz-Agüero y Galván, 1992; Danemann y De La Cruz -Agüero, 1993; De La Cruz-Agüero et al., 1994, 1996; Rosales-Casián, 1996 y De La Cruz-Agüero y Cota, 1998), como del sur (Ramírez et al., 1964; Ramírez y Páez, 1965; Yáñez-Arancibia, 1980; Coronado y Amezcua-Linares, 1988; Madrid et al., 1997, 1998 y Tapia-García et al., 1998). Sin embargo, los trabajos realizados en el Pacífico central mexicano son escasos y en particular los del estado de Nayarit que se refieren a la ictiofauna de manglar y de la plataforma interna (Amezcua-Linares, 1987; 1996; García-

Recibido: 04 octubre 2005; aceptado: 13 abril 2006
Ramírez y Lozano-Vilano, 1992).

Los litorales de los estados de Nayarit y Jalisco, específicamente en Bahía de Banderas y zonas adyacentes, albergan una gran diversidad de especies de interés científico, recreativo y comercial (ballena jorobada, tortugas marinas, peces de pico, pelágicos menores, cabrillas, pargos y otras), relacionada con la amplia variedad de hábitat disponibles, tales como zonas someras de playas arenosas, desembocaduras de ríos, áreas de litoral pedregoso, acantilados, zonas con arrecifes coralinos, así como de aguas abiertas.

Con el objeto de tener un análisis adecuado de la ictiofauna de esta región, entre 1997 y 1998, personal del Centro Regional de Investigación Pesquera Bahía de Banderas (CRIP-BB-INP), localizado en la Cruz de Huanacaxtle, Nayarit, dependiente del Instituto Nacional de Pesca-SAGARPA, llevó a cabo un programa de investigación sobre este particular. Las recolectas realizadas para establecer el conocimiento taxonómico de nivel alfa de 
una región y la conformación de sus elencos sistemáticos, constituyen el punto de partida para subsecuentes estudios y análisis, tales como las de tipo pesquero, manejo de recursos, evaluación ambiental, además de estudios filogenéticos y biogeográficos, entre otros. De manera adicional estos trabajos, apoyados en colecciones de referencia y con el respaldo en bases de datos, presentan una utilidad y aplicación discutida por De La Cruz-Agüero et al. (1994, 1996), además de apoyar la confiabilidad de los estudios y análisis citados. Esta contribución constituye la primera lista sistemática de la ictiofauna de Bahía de Banderas y áreas adyacentes.

\section{Materiales y métodos}

Bahía de Banderas y su zona litoral aledaña, se ubica en el Pacífico central mexicano y la comparten los estados de Nayarit y Jalisco. Se localiza entre $20^{\circ} 07^{\prime}$ y $21^{\circ} 08^{\prime}$ N y $105^{\circ} 10^{\prime}$ y $105^{\circ} 45^{\prime}$ O (Fig. 1). La bahía alberga una biodiversidad influenciada por los extremos septentrional y meridional de varias masas de agua: al norte, la Corriente de California y el agua subtropical del reflujo del Golfo de California y por el sur, la masa de agua tropical proveniente de los sistemas de corrientes Norecuatorial y de Costa Rica (Wyrtki, 1965). Se ubica en la región zoogeográfica del Pacífico oriental (Moyle y Cech, 1988), dentro de la Provincia Mexicana y queda incluida en la boca del Golfo de California, importante subprovincia oceanográfica: (Briggs, 1974).

La Bahía de Banderas es una de las más grandes del mundo, con extensión aproximada de $4289 \mathrm{~km}^{2}$, anchura promedio de $32 \mathrm{~km}$ (norte-sur) y longitud de casi $42 \mathrm{~km}$ (este-oeste). La costa norte se localiza en el estado de Nayarit y se extiende desde Punta Mita, la terminación de la Sierra Vallejo-Talpa, hasta la boca del río Ameca. Las áreas al sur del Ameca y a lo largo de la costa sur se ubican en el estado de Jalisco, con sus límites en Cabo Corrientes, que se configura por las estribaciones de la Sierra Chamela-Cabo Corrientes.

El área de la cuenca presenta la influencia del río Ameca, que se une al río Mascota para después desembocar en la parte central de la bahía. Existen otros ríos y arroyos como: el Pitillal, los Camarones, el Cuale, Mismaloya, Nogalitos, Palo María, Tomatlán, Tuito y Tabo, que desembocan en la bahía. Dentro de sus márgenes se presenta como un elemento fisiográfico característico el estero El Salado; mientras que en su interior en dirección noreste, se localizan las Islas Marietas. Este complejo insular está separado de Punta Mita por un canal cuya longitud aproximada es de 6.5 $\mathrm{km}$, profundidad menor a $45 \mathrm{~m}$ y su distancia desde Puerto Vallarta es de $32 \mathrm{~km}$; se conforma por dos islas grandes y una pequeña, así como algunas rocas aisladas (Zweifel, 1960).

El tipo de mareas en Bahía de Banderas es mixto y la circulación que predomina es hacia el noroeste con una

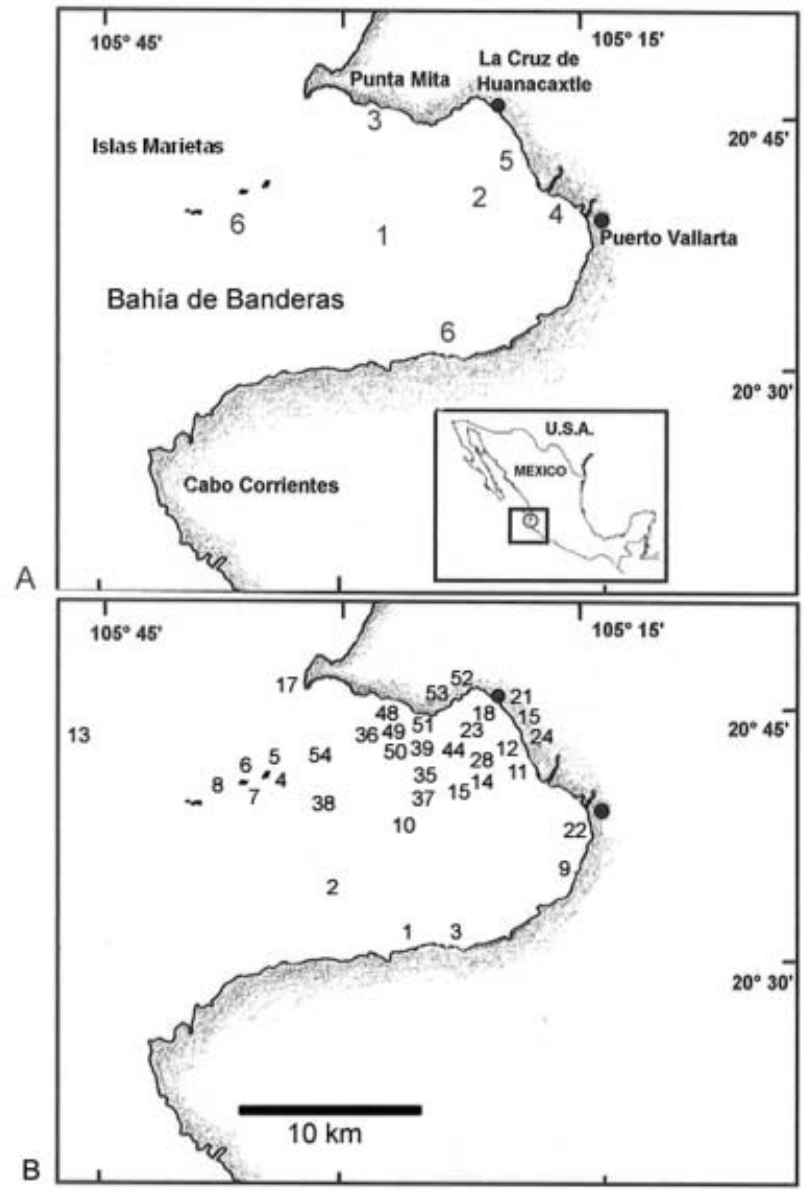

Figura 1. A. Localización geográfica y tipos de fondos y playas de la Bahía de Banderas: 1. Fondo rocoso; 2. Fondo arenoso; 3. Playa rocosa; 4. Estero; 5. Playa arenosa; 6. Arrecife. B. Ubicación de los diferentes sitios de muestreo. Cada numeral corresponde a las estaciones de recolecta, se omiten aquellos sobrepuestos por la escala (véase texto y Cuadro 1).

velocidad de 1 a 1.3 nudos con salida por la parte sur. La plataforma continental es bastante reducida y su área muy pequeña, además de lo escarpado del relieve submarino; su declive es muy acentuado y se encuentran zonas bastante profundas cerca de la línea de costa. La máxima profundidad de la bahía es mayor de $1000 \mathrm{~m}$ y la media es de $300 \mathrm{~m}$.

En la Bahía de Banderas se aprovechan los distintos recursos pesqueros ("escama") de tipo artesanal, cuya captura se desarrolla a lo largo del año sobre especies de serránidos y lutiánidos (cabrillas y pargos) y, en menor medida, guerreidos, paralíctidos y hemúlidos (mojarras, lenguados y burritos). Además, de acuerdo con la temporada, se pescan diversas especies de tiburón (carcarínidos y esfírnidos), así como peces vela y marlínes (istiofóridos). Por otra parte en este cuerpo de agua y áreas adyacentes, se llevan a cabo actividades de ecoturismo y conservación como: observación de la ballena jorobada y aves marinas, torneos de pesca deportiva, campamentos tortugueros y buceo guiado en área 
arrecifales.

Las recolectas se realizaron en 54 sitios preestablecidos, con base en el perfil batimétrico de la bahía, lo cual determinó que la mayor parte de estos sitios se ubicaran en la zona norte (Fig. 1B y Cuadro 1). Las capturas se hicieron de manera sistemática, en los distintos hábitats que caracterizan a la bahía, como: áreas de afluentes, playas arenosas, playas rocosas y arrecifes coralinos (Fig. 1A) y en diferentes niveles de profundidad, utilizándose las artes propias de la pesca comercial así como redes de prueba.

Las artes de pesca se utilizaron en un ciclo anual, que comprendió de marzo de 1997 a abril de 1998. El tipo y su frecuencia de uso fue la siguiente: barco camaronero de manera semestral (abril y septiembre de 1997); red de arrastre tipo chango en forma bimestral; chinchorro de arrastre playero (100 m de longitud) capturas bimestrales; redes agalleras (de 50 a $500 \mathrm{~m}$ de longitud), palangres tiburoneros y palangres para pargos de manera estacional (abril, julio, octubre de 1997 y enero de 1998). Asimismo, las fisgas y líneas de mano para pesca de fondo o curricán se utilizaron en cada estación del año. También se realizaron actividades mensuales de pesca exploratoria por medio de cuatro modelos básicos de trampas menores (nasas): plegables de forma rectangular, apilables de forma de rombo, tipo canasta redonda y apilables de forma cónico-truncada. La prospección se hizo a profundidades de 50 a 500 metros. Para la zona arrecifal, se llevaron a cabo muestreos bimestrales utilizando técnicas visuales rápidas, que consisten tanto en la identificación como en la cuantificación de los peces mientras se realiza el buceo en dos transectos por estación y un cuadrante de 5 X $5 \mathrm{~m}$.

Todo el material recolectado o bien una muestra representativa, se fijó en el campo con formalina comercial al $10 \%$ neutralizada con bórax (Lagler et al., 1984). Los ejemplares se depositaron en bolsas de plástico y cubetas etiquetadas con datos de fecha, hora, arte y sitio de recolecta. En el laboratorio se identificaron los ejemplares mediante las claves y descripciones de Jordan y Evermann (18961900), Meek y Hildebrand (1923-1928), Hildebrand (1946), Castro-Aguirre (1965, 1978), Instituto Nacional de Pesca (1976), Eschmeyer et al. (1983), Goodson (1988), Gotshall (1989), Fisher et al. (1995), Castro-Aguirre y Espinosa-Pérez (1996), De La Cruz-Agüero et al. (1997), Castro-Aguirre et al. (1999) y Thomson et al. (2000). Por último, los materiales biológicos se conservaron en una dilución de alcohol etílico al $70 \%$.

Para fines de interpretación del listado sistemático, los ejemplares de las especies recolectadas y depositadas en la colección ictiológica del CRIP-BB-INP, presentan sus nombres precedidos por un guión. Las que sólo fueron observadas y en alguna ocasión fotografiadas, pero para las que no se contó con información suficiente para su catalogación, sus nombres se presentan sin simbología. El ordenamiento sistemático para las categorías supragenéricas se realizó de acuerdo a Nelson (1994), con varias modificaciones para las rayas y especies afines según CastroAguirre y Espinosa Pérez (1996) y McEachran y Aschliman (2004) y de los tiburones según Espinosa Pérez et al. (2004). La clasificación de los géneros y sus respectivas especies se realizó en orden alfabético y en la nomenclatura de las especies se mantuvieron los criterios de Eschmeyer (1998) y Nelson et al. (2004)

En la lista sistemática, el número de catálogo de la colección del CRIP-BB-INP, se presenta entre corchetes [ ], el número de especimenes para dicho registro entre paréntesis ( ) y el arte de pesca con que fueron capturados entre llaves \{\}. Para esta última información se sigue la siguiente denominación: $\mathrm{AG}=$ red agallera; $\mathrm{AL}=$ almadraba; $\mathrm{AT}=$ atarraya; $\mathrm{CA}=$ barco camaronero; $\mathrm{CP}=$ caña de pescar; $\mathrm{CHA}=$ chango tipo camaronero; $\mathrm{CHR}=$ chinchorro de playa; $\mathrm{FO}=$ fotografía; $\mathrm{HA}=$ arpón hawaiano; $\mathrm{LA}=$ línea de mano y anzuelo; $\mathrm{MA}=$ manualmente; $\mathrm{OB}=$ observado; $\mathrm{PA}=$ palangre; $\mathrm{RC}=$ red de cuchara $\mathrm{TR}=$ trampa; $\mathrm{VA}=$ varado $\mathrm{y}$ $\mathrm{VI}=$ video. Adicionalmente, se anota en negritas el intervalo de tallas, expresado en centímetros de longitud total, de todos los individuos en la colección junto con los procesados en el campo.

Por último y de acuerdo con los intervalos de distribución conocidos, con letras mayúsculas se describe la afinidad zoogeográfica de acuerdo al esquema propuesto por Briggs (1974): PS= Provincia de San Diego; PC $=$ Provincia de Cortés (sinuscaliforniana); $\mathrm{PM}=$ Provincia Mexicana; $\mathrm{PP}=$ Provincia Panámica; T= amplia distribución en el Pacífico oriental; adicionando con letras minúsculas y en cursivas, el hábitat de recolecta en los siguientes términos: playa arenosa $=p a$; playa $\operatorname{rocosa}=p r$; fondo $\operatorname{arenoso}=f a$; fondo rocoso $=f r$ (las de litoral y de profundidad respectivamente); arrecife $=a r$; oceánica $=$ oc y estero= es. El signo “?”, implica información desconocida, no consignada en bitácoras o extraviada. Aquellas especies precedidas de un asterisco, representan nuevos registros o extensiones de distribución para el área de estudio.

\section{Resultados}

A partir de las recolectas en Bahía de Banderas, se integró una lista con un total de 74 familias, 145 géneros y 210 especies. Las familias mejor representadas son: Carangidae (19), Haemulidae (16), Sciaenidae (13), Serranidae (11) y Paralichthyidae (8). Un total de 69 familias, 140 géneros y 205 especies fueron recolectadas y depositadas en la Colección Ictiológica del CRIP-BB-INP y 5 sólo fueron observadas.

Los especimenes recolectados presentaron un intervalo de tallas de longitud de $1.6 \mathrm{~cm}$ (Bathygobius ramosus) a $229 \mathrm{~cm}$ (Makaira indica). Las especies Eucinostomus dowii, Chromis atrilobata y Lutjanus peru resultaron las de mayores abundancias durante el período de estudio.

Labase de datos de los peces recolectados y su información 
Cuadro 1. Sitios de recolecta de peces en la Bahía de Banderas y zonas adyacentes, con sus respectivas coordenadas geográficas y las artes de pesca empleadas durante el periodo 1997-1998.

1. Sur de Bahía de Banderas

2. Suroeste de Bahía de Banderas

3. Majahuitas

4. Marietas (Ojo de buey)

5. Marietas (Isla Larga)

6. Marietas (Isla Redonda e Isla Larga)

7. Marietas (La Cueva del Muerto)

8. Marietas (NW de Isla Redonda)

9. Playa Los Muertos Puerto Vallarta

10. Palito Verde

11. Campamento tortuguero

12. Restaurante "Etc"

13. La Corbeteña

14. Punta Tecuchitán

15. El Edén

16. El Tizate

17. El Anclote Punta de Mita

18. Bucerías frente a la plaza

19. Entre Bucerías y La Cruz

20. Bucerías a lo largo del pueblo

21. Flamingos ( $\mathrm{N}$ de Nuevo Vallarta)

22. La Marina Nuevo Vallarta

23. Playas de Nuevo Vallarta

24. El Palmar Nuevo Vallarta

25. Entre La Cruz y Bucerías

26-50. Arrastres camaroneros

en la Bahía de Banderas (lances 1- 25)

51. La Cruz de Huanacaxtle

52. La Cruz de Huanacaxtle

53. La Cruz de Huanacaxtle (muelle)

54. Frente a La Cruz de Huanacaxtle $20^{\circ} 30^{\prime} 18^{\prime \prime}-105^{\circ} 26^{\prime} 07^{\prime \prime}$

$20^{\circ} 34^{\prime} 12^{\prime \prime}-105^{\circ} 30^{\prime} 08^{\prime \prime}$

$20^{\circ} 30^{\prime} 30^{\prime \prime}-105^{\circ} 23^{\prime} 48^{\prime \prime}$

$20^{\circ} 39^{\prime} 54^{\prime \prime}-105^{\circ} 32^{\prime} 55^{\prime \prime}$

$20^{\circ} 41^{\prime} 16^{\prime \prime}-105^{\circ} 33^{\prime} 24$ "'

$20^{\circ} 41^{\prime} 55^{\prime \prime}$ - $105^{\circ} 34^{\prime} 28^{\prime \prime}$

$20^{\circ} 41^{\prime} 49^{\prime \prime}-105^{\circ} 34^{\prime} 55^{\prime}$ "

$20^{\circ} 42^{\prime} 21^{\prime \prime}-105^{\circ} 34^{\prime} 07^{\prime}$ '

$20^{\circ} 35^{\prime} 02$ " - $105^{\circ} 15^{\prime} 19$ "'

$20^{\circ} 42^{\prime}$ 0" $-105^{\circ} 24^{\prime} 36^{\prime \prime}$

$20^{\circ} 42^{\prime} 02^{\prime \prime}-105^{\circ} 17^{\prime} 59$ "

$20^{\circ} 42$ ' 30" - 105 19' 37'"

$20^{\circ} 43^{\prime} 0 "$ - $105^{\circ} 50$ ' 0"

$20^{\circ} 43^{\prime} 28^{\prime \prime}-105^{\circ} 25^{\prime} 09$ "

$20^{\circ} 43^{\prime} 57^{\prime \prime}$ - 105 19' 07'”

$20^{\circ} 44^{\prime} 44^{\prime \prime}-105^{\circ} 22^{\prime} 17$ "'

$20^{\circ} 45^{\prime} 15^{\prime \prime}-105^{\circ} 32$ ' 30"

$20^{\circ} 44^{\prime} 14^{\prime \prime}$ - $105^{\circ} 19^{\prime} 44^{\prime \prime}$

$20^{\circ} 43$ ' 16" - $105^{\circ} 20$ ' 9"

$20^{\circ} 43^{\prime} 45^{\prime \prime}$ - $105^{\circ} 19^{\prime} 52^{\prime \prime}$

$20^{\circ} 44^{\prime} 17^{\prime \prime}$ - 105 19'34"

$20^{\circ} 40^{\prime} 59^{\prime \prime}-105^{\circ} 17^{\prime} 25^{\prime \prime}$

$20^{\circ} 44^{\prime} 02^{\prime \prime}-105^{\circ} 20^{\prime} 43^{\prime \prime}$

$20^{\circ} 43^{\prime} 29^{\prime \prime}-105^{\circ} 18$ ' 37"

$20^{\circ} 44^{\prime} 01$ ' - $105^{\circ} 22^{\prime} 13^{\prime \prime}$

$20^{\circ} 41^{\prime} 30^{\prime \prime}$ - $105^{\circ} 20^{\prime} 17^{\prime \prime}$

$20^{\circ} 44^{\prime} 48^{\prime \prime}$ - $105^{\circ} 22^{\prime} 47^{\prime \prime}$

$20^{\circ} 44^{\prime} 10^{\prime \prime}-105^{\circ} 23$ ' 12 "

$20^{\circ} 44^{\prime} 30^{\prime \prime}-105^{\circ} 21^{\prime} 59^{\prime \prime}$

$20^{\circ} 44^{\prime} 48^{\prime \prime}$ - $105^{\circ} 22^{\prime} 47^{\prime \prime}$

$20^{\circ} 40^{\prime} 49^{\prime \prime}-105^{\circ} 30^{\prime} 57^{\prime}$ '
Trampas nasas y palangre

Trampas nasas y palangre

Visual (buceo)

Red cuchara anzuelos

Red cuchara

Red cuchara

Visual (buceo)

Red cuchara

Trampas nasas y palangre

Red agallera (200 m)

Chinchorro (100 m)

Chinchorro (100 m)

Chinchorro (100 m)

Línea de mano

Red de arrastre (20 m)

Red agallera (200 m)

Manual

Red de arrastre (6 m)

Red de arrastre (21 m)

Red de arrastre (10 m)

Chinchorro (100 m)

Manual

Red de arrastre (15 m)

Chinchorro (100 m)

Barco camaronero

Barco camaronero

Barco camaronero

Almadraba

Red de arrastre (18 m)

Línea de mano

Línea de mano accesoria, se desarrolló bajo un modelo relacional (por ejemplo: MSAccess) siguiendo los lineamientos que para los fines ha establecido la Comisión Nacional para el Conocimiento y uso de la Biodiversidad (CONABIO).
La información curatorial de este acervo, forma parte del Sistema Nacional de Información de Biodiversidad (SNIBCONABIO) en cuyo sitio de Internet (www.conabio.gob.mx) está disponible para su consulta. 
Listado sistemático:

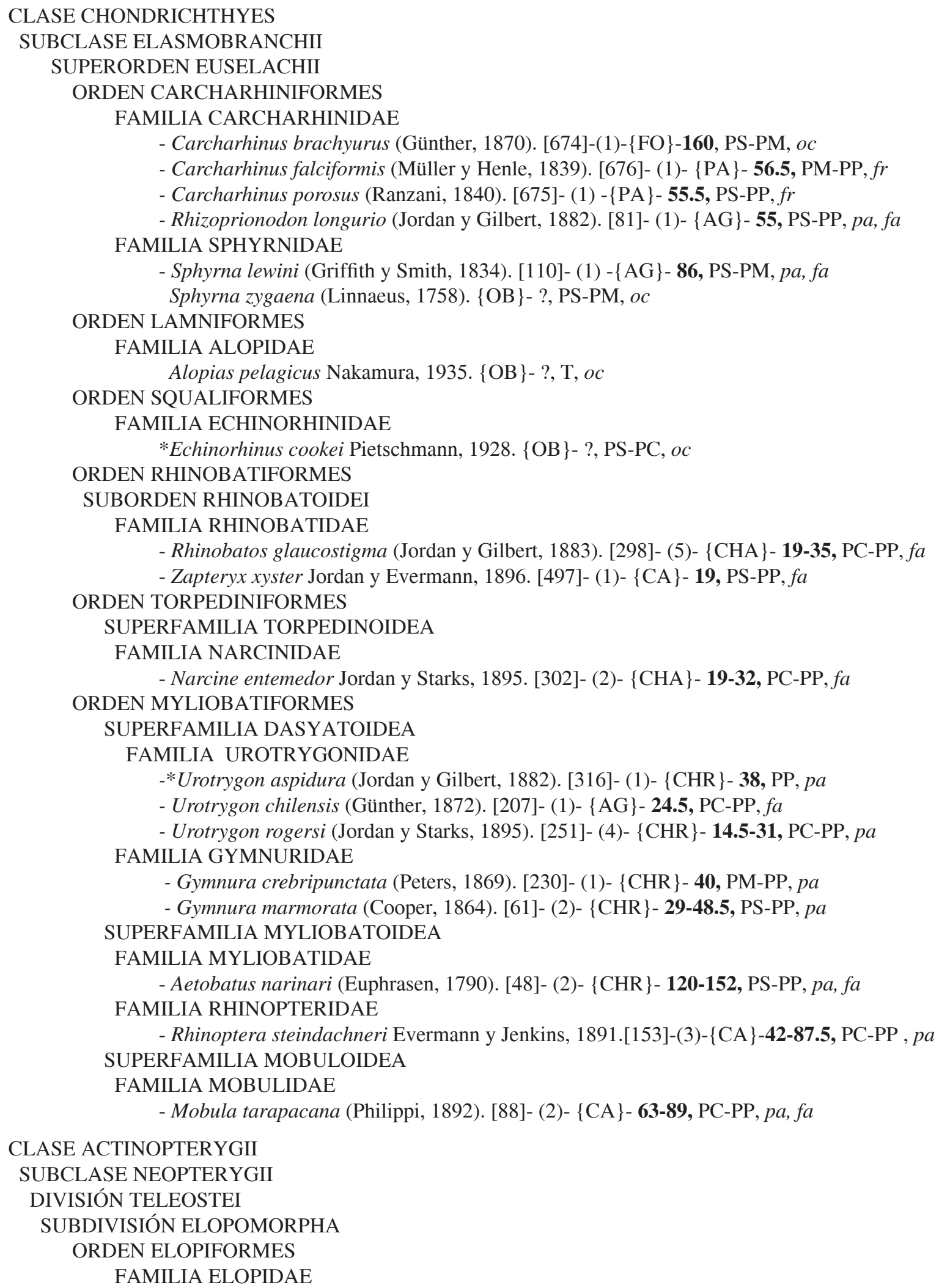


- Elops affinis Regan, 1909. [155]- (5)- \{CHR \}- 22.5-50, PS-PP, pa

ORDEN ALBULIFORMES

FAMILIA ALBULIDAE

-Albula nemoptera (Fowler, 1911). [620]- (7)- \{AL \}- 25-31, PM-PP, fr

- Albula (sp. aff. esuncula Garman, 1899). [631]- (4)- \{CHA\}- 25-32, PS-PP, fa

ORDEN ANGUILLIFORMES

SUBORDEN MURAENOIDEI

FAMILIA MURAENIDAE

- Gymnomuraena zebra (Shaw, 1797). [681]- (1)- \{VI\}- ?, PC-PP, ar

- Gymnothorax equatorialis (Hildebrand, 1946). [312]- (1)- \{PA\}- 54.2, PM-PP, ar, fr

SUBORDEN CONGROIDEI

FAMILIA OPHICHTHIDAE

- Myrichthys aspetocheiros McCosker y Rosenblatt, 1993. [692]-(1)-\{MA\}-34.5, PM-PP, pr

- Ophichthus zophochir Jordan y Gilbert, 1882. [162]- (2)- \{VA\}- 59-62.5, PS-PP, pr

FAMILIA MURAENESOCIDAE

SUBDIVISIÓN CLUPEOMORPHA

- Cynoponticus coniceps (Jordan y Gilbert, 1882). [311]- (3)- \{PA\}- 90-95, PS-PP, fr

\section{ORDEN CLUPEIFORMES}

SUBORDEN CLUPEOIDEI

FAMILIA ENGRAULIDAE

- Anchoa ischana (Jordan y Gilbert, 1882). [455]- (1)- \{CA \}- 12.5, PC-PP, fa

- Cetengraulis mysticetus (Günther, 1867). [29]- (3)- \{AG\}- 14-18, PM-PP, oc, fa

FAMILIA PRISTIGASTERIDAE

- Opisthopterus dovii (Günther, 1868). [263]- (2)- \{CHA\}- 18.5-22.5, PC-PP, fa

- Pliosteostoma lutipinnis (Jordan y Gilbert, 1882). [604]- (1)- \{CHR\}- 6.5, PM-PP, pa

FAMILIA CLUPEIDAE

- Opisthonema libertate (Günther, 1867). [106]- (23)- \{AG\}- 20-28.5, PM-PP, oc, fa

SUBDIVISIÓN EUTELEOSTEI

SUPERORDEN OSTARIOPHYSI

ORDEN SILURIFORMES

FAMILIA ARIIDAE

- Ariopsis platypogon (Günther, 1864). [107]- (12)- \{AG\}- 27.5-31, PS-PP, es, fa

- Ariopsis seemanni (Günther, 1864). [315]- (3)- \{CHR \}- 27, PM-PP, pa

- Bagre panamensis (Gill, 1863). [199]- (1)- \{AG\}- 35, PS-PP, es, fa

- Cathorops fuerthii (Steindachner, 1877). [190]- (2)- \{AG\}- 23-29, PM-PP, es, fa

SUPERORDEN CYCLOSQUAMATA

ORDEN AULOPIFORMES

SUBORDEN ALEPISAUROIDEI

FAMILIA SYNODONTIDAE

-*Synodus scituliceps Jordan y Gilbert, 1881. [309]- (2)- \{CHA\}- 26.2-33, PS-PC, fa

SUPERORDEN LAMPRIDIOMORPHA

ORDEN LAMPRIDIFORMES

FAMILIA REGALECIDAE

- Regalecus sp. ?- ?- $\{\mathrm{VA}\}-$ ?, PS-PP, fr

SUPERORDEN PARACANTHOPTERYGII

ORDEN OPHIDIIFORMES

SUBORDEN OPHIDIOIDEI

FAMILIA OPHIDIIDAE

- Cherublemma emmelas (Gilbert, 1890). [3]- (6)- \{TR \}- 17.5-24.5, PC-PP, fr

- Lepophidium prorates (Jordan y Bollman, 1890). [495]- (3)- \{CA $\}$ - 18.5-21, PC-PP, fa

-*Ophidion galeoides (Gilbert, 1890). [577]- (1)- \{CA \}- 17.5, PC-PP fa

ORDEN BATRACHOIDIFORMES

FAMILIA BATRACHOIDIDAE 
-*Porichthys analis Hubbs y Schultz, 1939 [421]- (3)- \{CA $\}$ - 15-22.5, PM, fa

ORDEN LOPHIIFORMES

SUBORDEN LOPHIOIDEI

FAMILIA LOPHIIDAE

- Lophiodes caulinaris (Garman, 1899). [615]- (1)- \{CHA $\}$ - 44.5, PS-PP, fa

SUBORDEN ANTENNARIOIDEI

FAMILIA ANTENNARIIDAE

- Antennarius avalonis Jordan y Starks, 1907. ?- ?- \{VA $\}$ - ?, PC-PP, ar

SUPERORDEN ACANTHOPTERYGII

SERIE MUGILOMORPHA

ORDEN MUGILIFORMES

FAMILIA MUGILIDAE

- Mugil cephalus Linnaeus, 1758. [43]- (31)- \{CHR \}- 38-45, PS-PP, es, pa

- Mugil curema Valenciennes, 1836. [14]- (11)- \{CHR \}- 32-34.5, PC-PP, es, pa, ar

SERIE ATHERINOMORPHA

ORDEN BELONIFORMES

SUBORDEN BELONOIDEI

FAMILIA BELONIDAE

- Tylosurus acus pacificus (Steindachner, 1876). [295]- (1)- \{LA\}- 91.5, T, oc, fr

- Tylosurus crocodilus fodiator Jordan y Gilbert, 1882. [294]- (2)- \{LA\}- 97-129, T, oc, fr

FAMILIA EXOCOETIDAE

- Fodiador acutus rostratus (Valenciennes, 1847). [637]- (4)- \{AL\}- 14.5-15, PS-PP, fr FAMILIA HEMIRAMPHIDAE

- Hyporhamphus naos Banford y Collette, 2001. [21]- (3)- \{AT $\}$ - 20-23, PS-PP, oc, fr

SERIE PERCOMORPHA

ORDEN BERYCIFORMES

SUBORDEN HOLOCENTROIDEI

FAMILIA HOLOCENTRIDAE

- Myripristis leiognathus Valenciennes, 1846. [679]- (1)- \{VI\}- ?, PC-PP, ar, pr

- Sargocentron suborbitalis Gill, 1863. [139]- (5)- \{AG\}- 10-18.5, PC-PP, ar, fr

ORDEN GASTEROSTEIFORMES

SUBORDEN SYNGNATHOIDEI

FAMILIA FISTULARIIDAE

- Fistularia commersonii Rüppell, 1838. [299]- (4)- \{CHA \}- 25.5-102, PM-PP, ar, fa

ORDEN SCORPAENIFORMES

SUBORDEN SCORPAENOIDEI

FAMILIA SCORPAENIDAE

- Scorpaena plumieri mystes (Jordan y Starks, 1895). [367]- (3)- \{CHA \}- 9-27, PM-PP ar, fa

- Scorpaena russula Jordan y Bollman, 1890. [463]- (2)- \{CA \}- 20-23, PM-PP, fa

FAMILIA TRIGLIDAE

- Bellator xenisma (Jordan y Bollman, 1890). [287]- (3)- \{CHA \}- 9-17.5, PS-PP, fa

- Prionotus ruscarius Gilbert y Starks, 1904. [429]- (4)- \{CA \}- 13.5, PM-PP, fa

- Prionotus stephanophrys Lockington, 1881. [459]- (3)- \{CA\}- 26-29, PS-PP, fa

ORDEN PERCIFORMES

SUBORDEN PERCOIDEI

FAMILIA CENTROPOMIDAE

- Centropomus armatus Gill, 1863. [633]- (1)- \{CHA $\}$ - 23.5, PM-PP, es, fa

- Centropomus medius Günther, 1864. [237]- (1)- \{CHR \}- 30, PM-PP, pa

- Centropomus nigrescens Günther, 1864. [94]- (5)- \{CHR \}- 35-41.5, PC-PP, pa

- Centropomus robalito Jordan y Gilbert, 1882. [231]- (1)- \{CHR \}- 28, PC-PP, es, pa

FAMILIA SERRANIDAE

- Alphestes mutiguttatus (Günther, 1867). [573]- (1)- \{CA \}- 13.5, PM-PP, fa

- Cephalopholis panamensis (Steindachner, 1877). [34]- (1)- \{HA \}- 25, PC-PP, ar, fr 
- Diplectrum labarum Rosenblatt y Johnson, 1974. [396]- (3)- \{MA\}- 9.5-26.5, PS-PP, pr

- Diplectrum pacificum Meek y Hildebrand, 1925. [418]- (17)- \{CA\}- 17-20, PS-PP, fa

- Epinephelus acanthistius (Gilbert, 1892). [464]- (2)- \{CA\}- 9.5-26, PS-PP, fa

- Epinephelus analogus Gill, 1863. [499]- (1)- \{CA\}- 21, PS-PP, fa

- Epinephelus itajara (Lichtenstein, 1822). [602]- (1)- \{CA \}- 4, PC-PP, fa

- Epinephelus labriformis (Jenyns, 1840). [137]- (1)- \{AG\}- 27.5, PC-PP, ar, fr

- Paralabrax loro Walford, 1936. [617]- (1)- \{CHA\}- 40, PM-PP, fa

- Paralabrax maculatofasciatus (Steindachner, 1868). [323]- (1)- \{LA\}- 15.5, PS-PM, fr

- Rypticus nigripinnis Gill, 1861. [636]- (1)- \{CHA\}- 18, PC-PP, fa

FAMILIA APOGONIDAE

- Apogon pacificus (Herre, 1935). [683]- (1)- \{VI\}- ?, PC-PP, ar

FAMILIA NEMATISTIIDAE

- Nematistius pectoralis Gill, 1862. [49]- (4)- \{CHR\}- 23-45, PS-PP, oc, pa

FAMILIA ECHENEIDAE

- Remora osteochir (Cuvier, 1829). [205]- (1)- \{MA\}- 13.5, PS-PP, oc

- Remora remora (Linnaeus, 1758). [1]- (1)- \{MA $\}$ - 19, PS-PP, oc

FAMILIA CORYPHAENIDAE

- Coryphaena hippurus Linnaeus, 1758. [203]- (1)- \{CP\}- 129, PS-PP, fr

FAMILIA CARANGIDAE

- Alectis ciliaris (Bloch, 1787). [392]- (1)- \{CHA\}- 3.5, PM-PP, fa

- Carangoides otrynter (Jordan y Gilbert, 1883). [125]- (3)- \{AG\}- 16.5-19.5, PC-PP, fr

- Carangoides vinctus (Jordan y Gilbert, 1881). [124]- (3)- \{AG\}- 27-29, PS-PP, fr

- Caranx caballus Günther, 1868. [318]- (1)- \{CHR \}- 21, PM-PP, pa

- Caranx caninus Günther, 1867. [159]- (4)- \{CHR\}- 26.5-50, PS-PP, pa

- Caranx sexfasciatus Quoy y Gaimard, 1825. [126]- (2)- \{AG $\}-$ 31.5-33, PM-PP, ar, fr

- Gnathanodon speciosus (Forsskäl, 1775). [130]- (3)- \{AG \}- 26.5-67, PM-PP, fa

- Chloroscombrus orqueta Jordan y Gilbert, 1883. [108]- (2)- \{AG\}- 17-22, PS-PP, fr

- Hemicaranx leucurus (Günther, 1864). [104]- (2)- \{AG \}- 25-32.5, PC-PP, fr

- Hemicaranx zelotes Gilbert, 1898. [173]- (1)- \{AG\}- 20.5, PC-PP, fr

- Oligoplites refulgens Gilbert y Starks, 1904. [216]- (1)- \{CHR\}- 25, PM-PP, pa

- Oligoplites saurus (Bloch y Schneider, 1801). [215]- (1)- \{CHR\}- 26, PS-PP, pa

- Selar crumenophthalmus (Bloch, 1793). [22]- (1)- \{AG\}- 22, PC-PP, fa

- Selene brevoortii (Gill, 1863). [174]- (3)- \{AG\}- 15-22, PC-PP, fa

- Selene oerstedii Lütken, 1880. [7]- (1)- \{CHR\}- 24.5, PC-PP, pa

- Selene peruviana (Guichenot, 1866). [127]- (9)- \{AG \}- 15.5-23, PS-PP, fa

- Seriola lalandi Valenciennes, 1833. [619]- (1)- \{LA\}- 31.5, PS-PP, fr

- Trachinotus paitensis Cuvier, 1832. [128]- (5)- \{AG\}- 20.6-21.2, PS-PP, fa

- Trachinotus rhodopus Gill, 1863. [142]- (3)- \{CHR\}- 16-23.4, PS-PP, ar, pa

FAMILIA LUTJANIDAE

- Hoplopagrus guentheri Gill, 1862. [208]- (2)- \{AG\}- 25-48, PS-PP, ar, fr

- Lutjanus argentiventris (Peters, 1869). [50]- (1)- \{CHR $\}-16$, PS-PP, ar, pa

- Lutjanus colorado Jordan y Gilbert, 1882. [689]- (3)- \{MA\}- 7.8-12.2, PS-PP, pr, fr

- Lutjanus guttatus (Steindachner, 1869). [136]- (4)- \{AG \}- 16-21, PM-PP, ar, fr

- Lutjanus novemfasciatus Gill, 1862. [688]- (3)- \{MA\}- 2.5-3.5, PS-PP, ar, pr, fr

- Lutjanus peru (Nichols y Murphy, 1922). [322]- (3)- \{LA\}- 14.5-23, PM-PP, fr

FAMILIA GERRREIDAE

-*Diapterus aureolus (Jordan y Gilbert, 1882). [341]- (3)- \{CHA $\}$ - 13-14, PP, fa

- Diapterus peruvianus (Cuvier, 1830). [10]- (3)- \{CHR $\}$ - 17-26.5, PC-PP, pa

- Eucinostomus dowii (Gill, 1863). [238]- (2)- \{CHA\}- 13.5-22.5, PS-PP, fa

- Eucinostomus currani Zahuaranec en Yáñez, 1980. [112]- (5)- \{AG\}- 2.6-23, PS-PP, fa

- Gerres cinereus (Walbaum, 1792). [138]- (2)- \{AG\}- 27-32, PM-PP, fr

FAMILIA HAEMULIDAE

- Anisotremus caesius (Jordan y Gilbert, 1882). [132]- (2)- \{AG\}- 20.5-21.5, PM-PP, fa

- Anisotremus dovii (Günther, 1864). [164]- (1)- \{AG \}- 19.5, PM-PP, fa 
- Anisotremus interruptus (Gill, 1862). [131]- (1)- \{AG \}- 31.5, PM-PP, fa

- Haemulon flaviguttatum Gill, 1862. [26]- (1)- \{AG\}- 24, PS-PP, ar, fa

- Haemulon maculicauda (Gill, 1862). [116]- (3)- \{AG\}- 20.5-22.5, PS-PP, fa

- Haemulon scudderi Gill, 1862. [105]- (1)- \{AG \}- 26, PM-PP, fa

- Haemulon steindachneri (Jordan y Gilbert, 1882). [11]- (2)- \{CHR $\}$ - 24.5-29.5, PC-PP, pa

- Haemulopsis elongatus (Steindachner, 1879). [119]- (2)- \{AG\}- 25-44, PM-PP, fr

- Haemulopsis leuciscus (Günther, 1864). [243]- (3)- \{CHA\}- 17-23, PC-PP, fa

- Microlepidotus brevipinnis (Steindachner, 1869). [355]- (1)- \{CHA\}- 18, PC-PP, fa

- Orthopristis chalceus (Günther, 1864). [118]- (2)- \{AG\}- 23.5-27, PC, fr

-*Orthopristis reddingi Jordan y Richardson, 1895. [196]- (3)- \{AG\}- 23.3-30, PC, fa

- Pomadasys bayanus Jordan y Evermann, 1898. [690]- (1)- \{MA\}- 8, PS-PP, pr

- Pomadasys panamensis (Steindachner, 1876). [270]- (4)- \{CHA $\}$ - 14-21.5, PC-PP, fa

- Xenichthys xanti Gill, 1863. [197]- (2) \{AG\}- 23, PS-PP, fa

FAMILIA SPARIDAE

- Calamus brachysomus (Lockington, 1880). [246]- (1)- \{CHA\}- 14.5, PS-PP, fa

FAMILIA POLYNEMIDAE

- Polydactylus approximans (Lay y Bennett, 1839). [183]- (2)- \{AG\}- 22.5-29.5, PS-PP, fa

- Polydactylus opercularis (Gill, 1863). [109]- (3)- \{AG\}- 22-31, PS-PP, fa

FAMILIA SCIAENIDAE

- Bairdiella icistia (Jordan y Gilbert, 1882). [101]- (2)- \{AG\}- 17.5-27, PC-PP, es, fa

-*Cynoscion reticulatus (Günther, 1864). [102]- (2)- \{AG \}- 30.5-38, PC, es, fa

- Cynoscion xanthulus Jordan y Gilbert, 1882. [236]- (1)- \{CHR\}- 46.5, PS-PP, pa

- Isopisthus remifer Jordan y Gilbert, 1882. [99]- (2)- \{AG\}- 15-21.5, PS-PP, fa

- Larimus acclivis Jordan y Bristol, 1898. [154]- (2)- \{CHR \}- 19.5-24, PC-PP, pa

- Larimus pacificus Jordan y Bollman, 1890. [69]- (2)- \{AG\}- 20.5-21, PM-PP, fa

-*Menticirrhus elongatus (Günther, 1864). [103]- (1)- \{AG \}- 35.5, PM, fa

- Menticirrhus paitensis Hildebrand, 1846. [149]- (1)- \{CHR \}- 30, PM-PP, pa

- Pareques viola (Gilbert, 1898). [20]- (1)- $\{\mathrm{RC}\}-7, \mathrm{PC}-\mathrm{PP}, a r, \mathrm{pr}$

- "Sciaena" sp. [184]- (3)- \{AG\}- 17-34, fa

- Umbrina bussingi López, 1980. [621]- (2)- \{PA\}- 22.5-23, PM-PP, fr

_*Umbrina wintersteeni Walker y Radford, 1992. [144]- (1)- \{CHR \}- 25.5, PS-PC, pa

- Umbrina xanti Gill, 1862. [100]- (3)- \{AG\}- 21.5-32, PM-PP, fa

FAMILIA MULLIDAE

- Mulloidichthys dentatus (Gill, 1862). [192]- (1)- \{AG\}- 28, PS-PP, ar, fa

- Pseudupeneus grandisquamis (Gill, 1863). [280]- (3)- \{CHR\}- 10.5-20, PS-PP, pa

FAMILIA CHAETODONTIDAE

- Chaetodon humeralis Günther, 1860. [442]- (5)- \{CA\}- 11-12.5, PS-PP, ar, fa

- Johnrandallia nigrirostris (Gill, 1862). [5]- (1)- \{RC \}- 10, PC-PP, ar, fr

FAMILIA POMACANTHIDAE

- Holacanthus passer Valenciennes, 1846. [66]- (1)- \{RC \}- 21, PM-PP, ar, fr

- Pomacanthus zonipectus (Gill, 1862). [65]- (3)- \{RC\}- 8-9 ,PM-PP, ar, fr

FAMILIA KYPHOSIDAE

- Kyphosus analogus (Gill, 1862). [641]- (1)- \{AL\}- 11, PS-PP, fr

- Kyphosus elegans (Peters, 1869). [24]- (1)- \{AG\}- 21, PC-PP, ar, fr

FAMILIA CIRRHITIDAE

- Cirrhitichthys oxycephalus (Bleeker, 1855). [682]- (1)- \{FO $\}$ - ?, PC-PP, ar, fr

- Cirrhitus rivulatus Valenciennes, 1846. [32]- (3)- \{HA\}- 22-26, PC-PP, ar, fr

SUBORDEN LABROIDEI

FAMILIA POMACENTRIDAE

- Chromis atrilobata Gill, 1862. [417]- (50)- \{OB \}- ?, PM-PP, ar

- Chromis limbaughi Greenfield y Woods, 1980. [677]- (1)- \{FO\}- ?, PC, ar

- Microspathodon dorsalis (Gill, 1862). [673]- (1)- \{HA\}- 27, PC-PP, ar

- Stegastes acapulcoensis (Fowler, 1944). [16]- (2)- \{RC $\}-5$, PM-PP, ar

- Stegastes flavilatus (Gill, 1862). [4]- (1)- \{RC\}- 5.5, PM-PP, ar, fr 


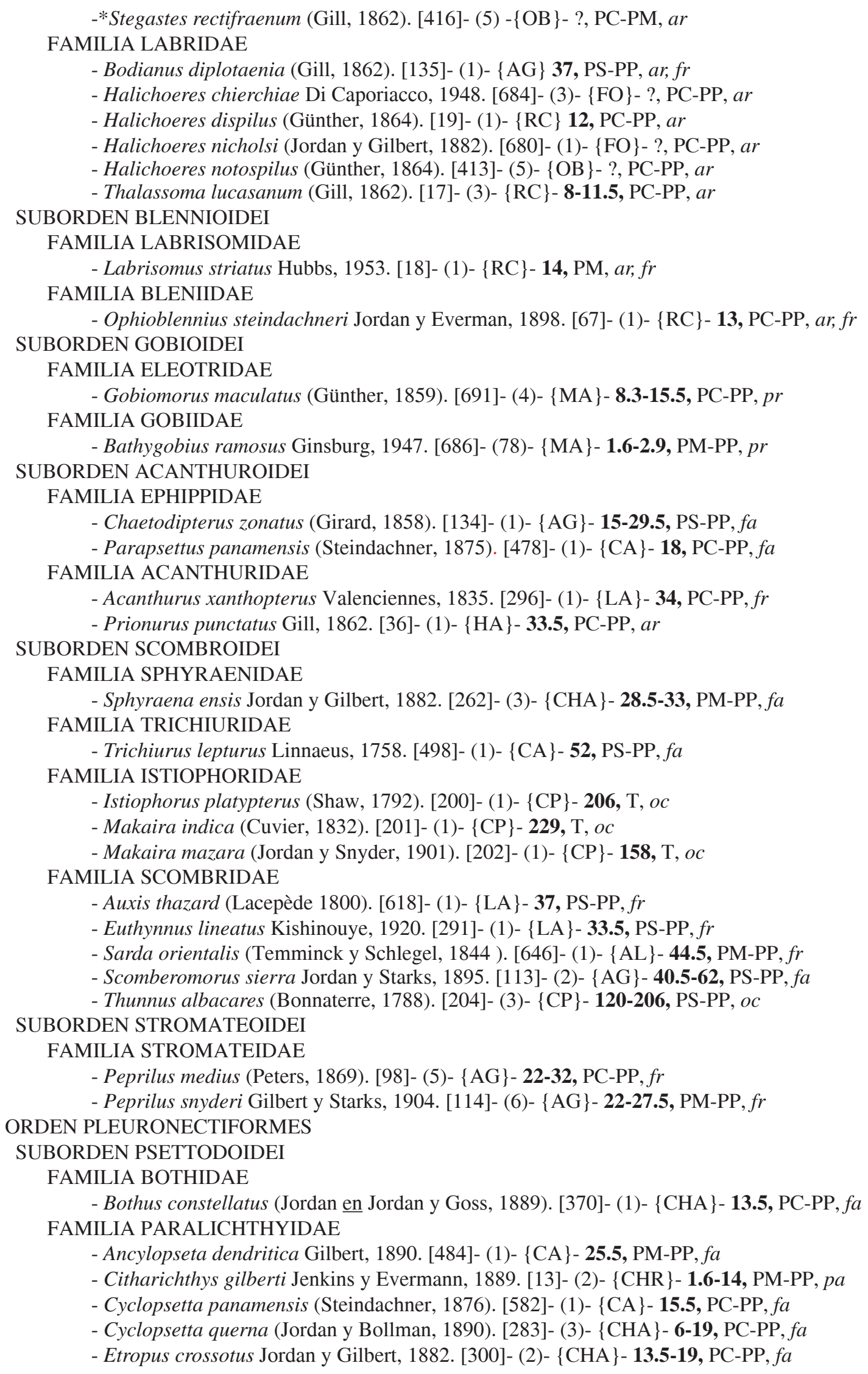


- Paralichthys woolmani Jordan y Williams, 1897. [133]- (1)- \{AG\}- 20, PS-PP, fa

- Syacium latifrons (Jordan y Gilbert, 1882). [31]- (1)- \{AG $\}-17$, PM-PP, fa

- Syacium ovale (Günther, 1864). [253]- (2)- \{CHA $\}$ - 12.5-18.5, PC-PP, fa

FAMILIA ACHIIRIDAE

- Achirus mazatlanus (Steindachner, 1869). [255]- (2)- \{CHA\}- 11-24,PS-PP, fa

- Achirus scutum (Günther, 1862). [371]- (1)- \{CHA\}- 12, PC-PP, fa

- Trinectes fonsecensis (Günther, 1862). [254]- (3)- \{CHA\}- 12.5-14, PS-PP, fa

FAMILIA CYNOGLOSSIDAE

- Symphurus atramentatus Jordan y Bollman, 1890. [259]- (4)- \{CHA \}- 16-18, PC-PP, fa

- Symphurus elongatus (Günther, 1868). [306]- (2)- \{CHA $\}$ - 17.5-19, PM-PP, fa

\section{ORDEN TETRAODONTIFORMES}

SUBORDEN TETRAODONTOIDEI

FAMILIA BALISTIDAE

- Balistes polylepis Steindachner, 1876. [37]- (3)- \{HA\}- 6-19, PS-PP, ar, fr

- Canthidermis maculata (Bloch, 1786). [408]- (1)- \{CHA\}- 52.5, PM-PP, fa

- Pseudobalistes naufragium (Jordan y Starks, 1895). [661]- (1)- \{CHA\}- 8.5, PM-PP, ar, fa

- Sufflamen verres (Gilbert y Starks, 1904). [292]- (4) \{LA\}- 23-31, PS-PP, ar, fr

FAMILIA MONACANTHIDAE

- Aluterus monoceros (Linnaeus, 1758). [642]- (2)- \{AL\}- 25.5, PC-PP, fr

FAMILIA OSTRACIIDAE

- Ostracion meleagris Shaw, 1796. [685]- (1)- \{FO\}- ?, PC-PP, ar

FAMILIA TETRAODONTIDAE

- Arothron meleagris (Lacepède, 1798). [678]- (1)- \{FO\}- ?, PC-PP, ar

- Canthigaster punctatissima (Günther, 1870). [6]- (1)- \{RC\} 5.5, T, ar, fr

- Lagocephalus lagocephalus (Linnaeus, 1758). [616]- (1)- \{CHA\} 22, PS-PP, fa

- Sphoeroides annulatus (Jenyns, 1842). [39]- (3)- \{CHR \} 16-35.5, PS-PP, pa

- Sphoeroides lobatus (Steindachner, 1870). [366]- (2)- \{CHA\} 7-15.5, PS-PP, fa

FAMILIA DIODONTIDAE

- Chilomycterus reticulatus (Linnaeus, 1758) [352]- (1)- \{CHA\} 42, PS-PP, fa

\section{(2)- $\{\mathrm{CHA}\}$ 22-33.7, T, ar, fa}

- Diodon hystrix Linnaeus, 1758 [241]

\section{Discusión}

Los resultados demuestran la presencia de una ictiodiversidad importante, si se le compara con la encontrada en otros ecosistemas similares. Así por ejemplo, de todos los sistemas costeros estudiados en la península de Baja California (De la Cruz Agüero et al., 1994; 1996; Rosales-Casián, 1996; De La Cruz Agüero y Cota, 1998) la riqueza de especies, géneros y familias de Bahía de Banderas es superior, con excepción de la bahía de La Paz en la cual se han registrado más de 400 especies (De La Cruz Agüero et al., 1997). No obstante, estos patrones comparativos pudieran estar influenciados por el esfuerzo de captura aplicado (tiempo, número de sitios y artes de pesca utilizados), superior para Bahía de Banderas.

Por otra parte, la topografía costera de toda la zona, la influencia de los sistemas de corrientes y la posición geográfica de la Bahía de Banderas, propician la existencia de una gran variedad de hábitats, lo que sin duda contribuye a la conformación de esta ictiodiversidad. De ahí que la comparación de esta región (central del Pacífico mexicano) con un "desierto" (Madrid et al., 1998) se debe al reducido número de investigaciones ictiológicas realizadas a la fecha. Lo anterior se corrobora, aunque de modo indirecto, con la cantidad aquí mencionada de nuevos registros y extensiones de ámbito para la zona.

El análisis de la información publicada (literatura no gris, sensu Collette, 1990) sobre el ámbito de distribución de las especies recolectadas, permite establecer que alrededor del 35\% (76 especies) habitan tanto la Región de California (sensu Briggs, 1974) en sus dos provincias: de San Diego (PS) y de Cortés (PC) como en la Región del Pacífico Oriental en sus provincias Mexicana (PM) y Panámica (PP). Siete especies se conocían sólo para las provincias de afinidad templado-cálida de la Región de California (PS + PC), constituyéndose de acuerdo con la literatura como extensiones de distribución y en algunos casos nuevos registros dentro de las provincias Mexicana o Panámica. El $26 \%$ (54) de las especies podrían considerarse como tropicales ya que su distribución actual sólo incluye las provincias PM y PP. Otras 63 especies (31\%) se pueden catalogar como de afinidad tropical (o de "transición tropical", distribuidas sólo en PC, PM y PP). Tres especies se podrían catalogar como de afinidad templada (o de "transición templada", distribuidas en PS, PC y PM), mismas que no se conocen al sur del Golfo 
de Tehuantepec. De modo adicional, seis son cosmopolitas y circumtropicales. Así, se puede concluir que al considerar la afinidad de la ictiofauna recolectada en Bahía de Banderas, el principal componente es tropical $(54+63=117$ especies; $57 \%$ ) y con una alta prevalencia de las que se conocen en toda la región del Pacífico oriental (sensu Briggs, 1974).

En esta contribución no se incluyeron a todas las especies crípticas de algunas familias (por ejemplo, Bleniidae, Chaenopsidae, Gobiesocidae, Gobiidae, Labrisomidae, Tripterygidae) que de acuerdo a las características o alcances de las recolectas y estudios en esta bahía, no se han analizado con detalle. En este sentido, se ha descrito que la Provincia Mexicana cuenta con endemismos en la familia Gobiesocidae (Briggs, 1955; 1960) y Clinidae (= [in part.] Labrisomidae) (Springer, 1958), por lo que es factible considerar que tal cifra se modificaría en caso de incluirlas.

Dentro de este contexto, 10 especies de las recolectadas: Urotrygon aspidura, Synodus scituliceps, Ophidion galeoides, Porichthys analis, Diapterus aureolus, Orthopristis reddingi, Cynoscion reticulatus, Menticirrhus elongatus, Umbrina wintersteeni y Stegastes rectifraenum y una de las observadas: Echinorhinus cookei, ven ampliada su área de distribución geográfica. En la revisión de los ámbitos de distribución de las especies sólo se consideraron como registros válidos, aquellos referidos en la literatura publicada de manera arbitrada.

La Bahía de Banderas, compartida por los estados de Nayarit y Jalisco, presenta un importante desarrollo social y económico actual, con tendencia de gran auge hacia el aprovechamiento de sus recursos y de modo particular en el ecoturismo. Si bien esta creciente iniciativa, en el sentido estricto de su definición, trata de conciliar el desarrollo económico con el equilibrio ecológico y humano, aún no se han establecido en la región las bases y pautas para su logro. Con todo, se identifica la gran importancia de la conservación de los recursos, como una actividad rentable, por medio de un manejo adecuado en su ámbito global e integral, para coadyuvar a beneficios duraderos y justos. A este respecto, el presente trabajo representa la primera contribución al conocimiento ictiofaunístico de este cuerpo costero, lo que podría servir para el fortalecimiento de otros estudios e investigaciones sobre dichos recursos.

\section{Agradecimientos}

Las recolectas de peces y la elaboración de la base de datos se realizaron con el apoyo financiero de la CONABIO (convenio FB459/L156/97). Se agradece el apoyo logístico y material otorgado por la Facultad de Ingeniería Pesquera de la Universidad Autónoma de Nayarit, en particular, al Ing. S. Castillo Vargas-Machuca, así como la colaboración especial del M. en C. J. L. Cervantes, de Eugenio y "El Sinaloa". Este trabajo no hubiera sido posible sin el apoyo de la maestra
Biól. S. Sánchez González y de los compañeros y colegas del CRIP-BB-INP: E. Martínez González, A. Solís Chávez, A. I. García Patiño, P. del Monte Luna, J. J. González Ruiz y S. Hernández Ventura. Los autores agradecen los apoyos de la COFAA-IPN y EDI-IPN.

\section{Literatura citada}

Amezcua-Linares, F. 1987. Dinámica y estructura de la comunidad de peces en un sistema ecológico de manglares de la costa del Pacífico de México, Nayarit. Anales del Instituto de Ciencias del Mar y Limnología, Universidad Nacional Autónoma de México 14: 221-248.

Amezcua-Linares, F. 1996. Peces demersales de la plataforma continental del Pacífico Central de México. Instituto de Ciencias del Mar y Limnología, UNAM-CONABIO, México, $184 \mathrm{p}$.

Briggs, J. C. 1955. A monograph of the clingfishes (order Xenopterygii). Stanford Ichthyological Bulletin 6: 1-224.

Briggs, JC, 1960. A new clingfish of the genus Gobiesox from the Tres Marías Islands. Copeia 3: 215-217.

Briggs, J. C. 1974. Marine zoogeography. McGraw-Hill, New York. 475 p.

Castro-Aguirre, J. L. 1965. Peces sierra, rayas, mantas y especies afines de México. Anales del Instituto Nacional de Investigaciones Biológico Pesqueras 1: 169-256.

Castro-Aguirre, J. L. 1978. Catálogo sistemático de los peces que penetran a las aguas continentales de México, con aspectos zoogeográficos y ecológicos. Serie Científica 19. Departamento de Pesca, México. xi + 298 p.

Castro-Aguirre, J. L., J. Arvizu M. y J. Páez B. 1970. Contribución al conocimiento de los peces del Golfo de California. Revista de la Sociedad Mexicana de Historia Natural 31: 107-181.

Castro-Aguirre, J. L. y H. Espinosa Pérez. 1996. Listados faunísticos de México. VII. Catálogo sistemático de las rayas y especies afines de México (Chondrichthyes: Elasmobranchii: Rajiformes: Batoideiomorpha). Instituto de Biología, Universidad Nacional Autónoma de México. México, D.F. 75 p.

Castro-Aguirre, J. L., H. Espinosa Pérez y J. J. SchmitterSoto. 1999. Ictiofauna estuarino-lagunar y vicaria de México. Noriega-Limusa, México, D.F., 705 p.

Collette, B. B. 1990. Problems with gray literature in fishery science. In Writing for fishery journals, J. Hunter (ed.). American Fisheries Society, Bethesda, Maryland. p. 27-31.

Coronado, M. C. y F. Amezcua-Linares. 1988. Distribución y abundancia de los peces demersales de la costa de Guerrero en el Pacífico de México. Anales del Instituto de Ciencias del Mar y Limnología, Universidad Nacional Autónoma de México 15: 67-94.

Danemann, G. D. y J. De La Cruz-Agüero. 1993. Ichthyofauna of San Ignacio Lagoon, Baja California Sur, México. 
Ciencias Marinas 19: 333-341.

De La Cruz-Agüero, J. y F. M. Galván. 1992. Peces mesopelágicos capturados en la costa occidental de Baja California Sur y Golfo de California: Cruceros Puma 1982-1988. Anales del Instituto de Ciencias del Mar y Limnología, Universidad Nacional Autónoma de México 19: 25-31.

De La Cruz-Agüero, J., F. M. Galván, A. C. Abitia, J. R. Rodríguez y F. J. Gutiérrez. 1994. Lista sistemática de los peces marinos de bahía Magdalena, Baja California Sur, México. Ciencias Marinas 20: 17-31.

De La Cruz-Agüero, J., M. M. Arellano y V. M. Cota. 1996. Lista sistemática de los peces marinos de las lagunas Ojo de Liebre y Guerrero Negro, B. C. S. y B.C., México. Ciencias Marinas 22: 111-128.

De La Cruz-Agüero, J., M. M. Arellano, V. M. Cota y G. De La Cruz-Agüero. 1997. Catálogo de los Peces Marinos de Baja California Sur. IPN - CONABIO. México, D.F. $346 \mathrm{p}$.

De La Cruz-Agüero, J. y V. M. Cota. 1998. Ictiofauna de la Laguna de San Ignacio, B.C.S., México: nuevos registros y ampliaciones de ámbito. Ciencias Marinas 24: $353-$ 358.

Eschmeyer, W. N. 1998. Catalog of fishes. California Academy of Sciences, San Francisco, California. 2095 p.

Eschmeyer, W. N., E. S. Herald y H. Hammann. 1983. A field guide to the Pacific coast fishes of North America. Hougthon Mifflin, Boston. 336 p.

Espinosa Pérez, H., J. L. Castro-Aguirre y L. Huidobro Campos. 2004. Listados faunísticos de México. IX. Catálogo sistemático de tiburones (Elasmobranchii: Selachimorpha). Instituto de Biología Universidad Nacional Autónoma de México. México, D.F. 134 p.

Fischer, W., F. Krupp, W. Schneider, C. Sommer, K. E. Carpenter y V. H. Niem. 1995. Guía FAO para la identificación de especies para los fines de la pesca, Pacífico Centro-Oriental, vols. II y III. FAO, Roma, p. 647-1812.

García-Ramírez, M. E. y M.L.Lozano-Vilano.1992. Lista revisada de los peces marinos costeros de Nayarit, Mexico. Publicaciones Biológicas F.C.B./U.A.N.L., México 6: 124-132.

Goodson, G. 1988. Fishes of the Pacific coast. Stanford University Press. Stanford, California. 267 p.

Gotshall, D. W. 1989. Pacific coast inshore fishes. Sea Challengers. Monterey, California. 96 p.

Hildebrand, S.F. 1946. A descriptive catalog of the shore fishes of Peru. Bulletin of the United States National Museum 189: 1-530.

Instituto Nacional de Pesca. 1976. Catálogo de peces marinos mexicanos. Secretaría de Industria y Comercio, México, D.F. 462 p.

Jordan, D. S. y B. W. Evermann. 1896-1900. The fishes of North and Middle America. Part. I (1896), II (1898), III (1898) \& IV (1900). Bulletin of the United States Natural
History Museum 47: 1-3313.

Lagler, K. F., J. E. Bardach, R. R. Miller. y D. R. M. Passino. 1984. Ictiología. AGT, México, D.F. 489 p.

Madrid, J. V., P. Sánchez y A. A. Ruiz. 1997. Diversity and abundance of a tropical fishery on the Pacific shelf of Michoacán México. Estuarine Coastal and Shelf Science 45: 485-495.

Madrid, J. V., A. L. Ruiz y B. I. Rosado. 1998. Peces de la plataforma continental de Michoacán y sus relaciones en el Pacífico mexicano. Revista de Biología Tropical 46: 267-276.

McEachran, J. D. y N. Aschliman. 2004. Phylogeny of Batoidea. In Biology of sharks and their relatives. J.C. Carrier, J. A. Musick y M.R. Heithaus (eds.). CRC Press, Boca Raton, Florida. p. 115-135.

Meek, S. E. y S. F. Hildebrand. 1923-1928. The marine fishes of Panama. Publications of the Field Museum of Natural History, Zoological Series 15: 1-1045.

Moyle, P. B. y J. J. Cech. 1988. Fishes an introduction to ichthyology. Prentice Hall, Englewood Cliffs, New Jersey. 559 p.

Nelson, J. S. 1994. Fishes of the world, 3a. edición. John Wiley, New York. 600 p.

Nelson, J. S., E. J. Crossman, H. Espinosa Pérez, L. T. Findley, C. R. Gilbert, R. N. Lea y J. D. Williams. 2004. Common and scientific names of fishes from the United States, Canada and, Mexico. 6a edición. American Fishery Society, Publicación Especial 29, Bethesda, Maryland. $386 \mathrm{p}$.

Ramírez H., G. Carrillo y D. Lluch B. 1964. Investigaciones ictiológicas en la costa de Chiapas. Lista de los peces colectados en las capturas camaroneras (agosto y septiembre 1959 y abril, mayo y junio 1960). Publicaciones Especiales del Instituto Nacional de Investigaciones Biológico Pesqueras 5: 1-17.

Ramírez H., E. y J. Arvizu M. 1965. Investigaciones ictiológicas en las costas de Baja California. I. Lista de peces marinos de Baja California colectados en el período 1961-1965. Anales del Instituto Nacional de Investigaciones Biológico Pesqueras 1: 293-324.

Ramírez H., E. y J. Páez B. 1965. Investigaciones ictiológicas en la costa de Guerrero. I. Lista de peces marinos de Guerrero colectados en el periodo 1961-1965. Anales del Instituto Nacional de Investigaciones Biológico Pesqueras 1: 325- 358.

Rosales-Casián, J. A. 1996. Ictiofauna de la bahía de San Quintín, Baja California, México, y su costa adyacente. Ciencias Marinas 22: 443-458.

Springer, V. G. 1958. Systematics and zoogeography of the clinid fishes of the subtribe Labrisomini Hubbs. Publications of Institution of Marine Science (University of Texas) 5: 417-492.

Tapia-García, M., C. Suárez-Nuñez, G. Cerdenares-Ladrón de Guevara, M. Macuilt-Montes y M. C. García-Abad. 1998. Composición y distribución de la ictiofauna del 
Mar Muerto, Pacífico Mexicano. Revista de Biología Tropical 46: 277-284.

Thomson, D. A., L. T. Findley y A. M. Kerstitch. 2000. Reef fishes of the sea of Cortez: The rocky-shore fishes of the Gulf of California. The University of Texas Press, Austin. 353 p.

van der Heiden, A. M. y L.T. Findley. 1988. Lista de los peces marinos del sur de Sinaloa, México. Anales del Instituto de Ciencias del Mar y Limnología, Universidad Nacional Autónoma de México 15: 209-224.
Wyrtki, K. 1965. Surfice currents in the eastern Equatorial Pacific Ocean. Inter-American Tropical Tuna Commission Bulletin 9: 270-304.

Yáñez-Arancibia, L. A. 1980. Taxonomía, ecología y estructura de las comunidades de peces en lagunas costeras con bocas efímeras del Pacífico de México. Publicaciones Especiales Centro de Ciencias del Mar y Limnología, Universidad Nacional Autónoma de México 2: 1-306.

Zweifel, R. 1960. Results of the Puritan-American Museum 